\title{
Murine and Chinese cobra venom-derived nerve growth factor stimulate chondrogenic differentiation of BMSCs in vitro: A comparative study
}

\author{
ZHIKANG MIAO ${ }^{1-4^{*}}$, ZHENHUI LU ${ }^{1-3^{*}}$, SHIXING LUO ${ }^{5 *}$, DANQING LEI $^{4}$, \\ YI HE ${ }^{1-3}$, HUAYU WU ${ }^{6}$, JINMIN ZHAO ${ }^{1-3,7}$ and LI ZHENG $^{1-4}$ \\ ${ }^{1}$ Guangxi Engineering Center in Biomedical Material for Tissue and Organ Regeneration; \\ ${ }^{2}$ Guangxi Collaborative Innovation Center for Biomedicine; ${ }^{3}$ Guangxi Key Laboratory of Regenerative Medicine, \\ ${ }^{4}$ Life Sciences Institute, Guangxi Medical University, Nanning, Guangxi 530021; ${ }^{5}$ Department of Orthopedics, \\ Ninth Affiliated Hospital of Guangxi Medical University, Beihai, Guangxi 536000; ${ }^{6}$ Department of Cell Biology and Genetics, \\ School of Premedical Sciences, Guangxi Medical University; ${ }^{7}$ Department of Orthopaedics Trauma and Hand Surgery, \\ The First Affiliated Hospital of Guangxi Medical University, Guangxi Medical University, \\ Nanning, Guangxi 530021, P.R. China
}

Received December 23, 2017; Accepted June 22, 2018

DOI: $10.3892 / \mathrm{mmr} .2018 .9307$

\begin{abstract}
Mesenchymal stem cell (MSC)-based therapy has been commonly used in cartilage reconstruction, due to its self-renewing ability and multi-differentiation potential. Nerve growth factor (NGF) from cobra venom has been reported to regulate chondrogenesis of bone-derived MSCs (BMSCs) and chondrocyte metabolism. Therefore, the present study aimed to determine whether other sources of NGF behave in the same manner as NGF from natural venom. The present study compared the effects of NGF from two sources, the commercially purchased recombinant murine $\beta$-NGF (mNGF) and cobra venom-derived NGF (cvNGF), on chondrogenesis of BMSCs by performing hematoxylin and eosin and fluorescein diacetate/propidium iodide staining, DNA and glycosaminoglycan quantization and reverse transcription-quantitative polymerase chain reaction to investigate cell morphology, viability, proliferation, glycosaminoglycan synthesis and cartilage-specific gene expression. The results demonstrated that cvNGF significantly accelerated cell proliferation and upregulated the expression of cartilage-specific genes,
\end{abstract}

Correspondence to: Professor Jinmin Zhao or Professor Li Zheng, Guangxi Engineering Center in Biomedical Material for Tissue and Organ Regeneration, Guangxi Medical University, 22 Shuangyong Road, Nanning, Guangxi 530021, P.R. China

E-mail: zhaojinmin@126.com

E-mail: zhengli224@163.com

*Contributed equally

Key words: nerve growth factor, bone-derived mesenchymal stem cells, chondrogenesis, Chinese cobra venom, recombinant murine $\beta$-nerve growth factor including aggrecan, SRY-box 9 and collagen type II $\alpha 1$ chain. Conversely, cvNGF reduced the expression levels of collagen type I $\alpha 1$ chain (a fibrocartilage marker), runt-related transcription factor 2 and enolase 2 compared with in the mNGF and control groups. In addition, Chinese cobra venom, which is the main resource of cvNGF, is abundant and inexpensive, thus greatly decreasing the cost. In conclusion, the present study demonstrated that cvNGF may be considered a potential growth factor for inducing chondrogenic differentiation of BMSCs.

\section{Introduction}

Articular cartilage has limited regenerative capabilities following injury, due to the lack of vascularization, reduced progenitor cell supply and the presence of few cells with low mitotic activity $(1,2)$. Cell-based therapies are considered useful approaches for cartilage restoration $(3,4)$. Mesenchymal stem cells (MSCs) can be isolated from multitudinous tissues, including bone marrow, adipose tissue and the placenta, and are frequently used as a source of cartilage restoration due to high yields, easy accessibility and the capability to differentiate along the chondrogenic lineage (5). Bone-derived MSCs (BMSCs) appear to be the most promising choice for cartilage regeneration, due to the ease with which they can be acquired, and their high proliferative capacity and chondrogenic differentiation ability (6). Growth factors, such as transforming growth factor- $\beta 1$, serve critical roles in the regulation the chondrogenic differentiation of MSCs. Although conditioned medium that regulates chondrogenesis of MSCs has been well established (7), the high cost, short half-life, versatility and functional heterogeneity of traditional growth factors remain an obstacle for stem cell-based therapy.

In our previous study, it was revealed that a novel growth factor, nerve grow th factor (NGF) extracted from Chinese 
cobra venom, specifically induces the chondrogenic differentiation of MSCs and further promotes cartilage repair (8). The chondral specificity of NGF is better than traditionally used growth factors, which may lead to osteophyte formation instead of chondrogenesis during cartilage regeneration (9-11). Other peptide neurotrophins, such as Nel-like molecule-1, which is abundantly secreted in neural tissue, are also reported to promote the proliferation of chondrocyte and maintain its phenotype in vitro (12). Grassel (13) also demonstrated the roles of sensory and sympathetic neurotransmitters on limb formation during the process of embryonic skeletal development. Notably, venom-derived NGF rarely induces BMSCs to differentiate into a neuronal phenotype (14).

There are numerous sources of NGF, which has been isolated from venom and body fluids, and formed by recombination. Lipps $(15,16)$ studied the bioactivity of NGF from bee, scorpion and spider venoms, and NGF from body fluids, including serum, saliva and urine, and compared these NGFs with cobra venom-derived NGF (cvNGF). The findings of these previous studies indicated that the biological activities of NGFs obtained from human body fluids on PC12 cells were minor in comparison to cvNGF, and the biological activity of bee NGF on PC12 cells was $1 / 10$ that of cvNGF. It remains to be determined as to whether NGF from other sources behaves the same as NGF extracted from natural venom.

The present study compared the effects of commercially purchased recombinant murine $\beta$-NGF (mNGF) and cvNGF on the chondrogenic differentiation of MSCs by detecting cell proliferation, glycosaminoglycan (GAG) synthesis and cartilage-specific gene expression. The findings of this study may aid in the clinical application of NGF for cartilage repair.

\section{Materials and methods}

Cell culture. BMSCs were isolated from bone marrow that was extracted from two male Sprague-Dawley rats (weight, 8-10 g; age, 3 days), which were purchased from the Animal Experimental Center of Guangxi Medical University (Nanning, China). The rats were housed at a constant temperature $\left(26^{\circ} \mathrm{C}\right)$ and relative humidity $(60 \%)$ under a reversed 12 -h dark/light cycle (lights on until 8:00 p.m.), with free access to a standard diet and water. All experiments were conducted in accordance with the standard guidelines approved by the Animal Care and Experiment Committee of Guangxi Medical University (Guangxi, China; protocol number: 2014-12-3), and the present study was approved by the ethics committee of Guangxi Medical University. Briefly, the rats were sacrificed with an injected overdose of pentobarbital, and bone marrow was collected from the bilateral femur by flushing with $\alpha$-modified Eagle's medium ( $\alpha$-MEM; Gibco; Thermo Fisher Scientific, Inc., Waltham, MA, USA) supplemented with $1 \%$ penicillin/streptomycin (Beijing Solarbio Science \& Technology Co., Ltd., Beijing, China). Following density gradient centrifugation $\left(1,100 \mathrm{x} \mathrm{g}\right.$ at $25^{\circ} \mathrm{C}$ for $\left.30 \mathrm{~min}\right)$, the isolated BMSCs were cultured in $\alpha$-MEM supplemented with 10\% fetal bovine serum (Gibco; Thermo Fisher Scientific, Inc.) and $1 \%$ penicillin/streptomycin. The culture medium was changed every 2 days. Characterization of isolated BMSCs was performed as described previously (5).
Cell seeding. Two sources of NGF were added to the cell cultures. mNGF was purchased from Peprotech, Inc. (Rocky Hill, NJ, USA), and cvNGF was extracted and purified from the venom of Chinese cobra (Naja naja atra) as described in our previous study (8). Briefly, BMSCs were cultured in chondrogenic medium including $50 \mu \mathrm{g} / \mathrm{ml}$ ascorbic acid (A7506; Sigma-Aldrich; USA), $100 \mathrm{nM}$ dexamethasone (D1756; Sigma-Aldrich; Merck KGaA, Darmstadt, Germany), 1\% insulin-transferrin-selenium solution (41400045; Gibco; Thermo Fisher Scientific, Inc.) and $0.06 \mu \mathrm{g} / \mathrm{ml} \mathrm{mNGF}$ or $6 \mu \mathrm{g} / \mathrm{ml}$ vNGF. For each experiment, cells at passage 2 were used and the seeding density was $2 \times 10^{4}$ cells $/ \mathrm{cm}^{2}$. Experiments were performed at 7,14 and 21 days.

Cytotoxicity assay. NGF toxicity on BMSCs was detected using the MTT assay (Gibco; Thermo Fisher Scientific, Inc.). BMSCs were seeded onto 96-well plates and treated with various concentrations of $\mathrm{mNGF}(0,0.0075,0.015,0.03,0.06$, $0.12,0.24,0.48$ and $0.96 \mu \mathrm{g} / \mathrm{ml})$ and $\operatorname{cvNGF}(0,1,1.5,2,3,4,6$, $8,12,16,24$ and 32 ) and incubated at $37^{\circ} \mathrm{C}$. After 3 days, $20 \mu \mathrm{l}$ MTT solution $(5 \mathrm{mg} / \mathrm{ml})$ was added and incubated at $37^{\circ} \mathrm{C}$ for $4 \mathrm{~h}$ in the dark. After removing the medium, the formazan crystals in each well were dissolved with $200 \mu$ l dimethyl sulfoxide (Gibco; Thermo Fisher Scientific, Inc.). The absorbance was measured at $570 \mathrm{~nm}$ using a microplate reader (Thermo Fisher Scientific, Inc.).

Cell viability assay. Cell viability was determined by fluorescein diacetate (FDA; GenWay Biotech, Inc., San Diego, CA, USA) and propidium iodide (PI; Sigma-Aldrich; Merck $\mathrm{KGaA}$ ) staining on days 7,14 and 21. Briefly, after rinsing with PBS for three times, $0.5 \mu \mathrm{M}$ FDA and $2 \mu \mathrm{M}$ PI in $1 \mathrm{ml}$ PBS was added to the cells and incubated in the dark for $5 \mathrm{~min}$. Images were captured under a laser scanning confocal microscope (Olympus Corporation, Tokyo, Japan).

Cell morphological examination. The BMSCs were seeded onto 24-well-plates and were cultured with $\mathrm{mNGF}(0.06 \mu \mathrm{g} / \mathrm{ml})$ or cvNGF $(6 \mu \mathrm{g} / \mathrm{ml})$ for 7, 14 and 21 days. The cells were then washed three times with PBS and fixed with $95 \%$ alcohol for $30 \mathrm{~min}$ at room temperature. Subsequently, the cells were washed with PBS and stained with $10 \%$ hematoxylin and 2\% eosin (H\&E; Nanjing Jiancheng Bioengineering Institute, Nanjing, China) at room temperature for $5 \mathrm{~min}$ and $30 \mathrm{sec}$, respectively. Finally, cells were observed and images were captured under an inverted phase contrast microscope (Olympus Corporation).

Cell proliferation analysis and biochemical assay. After being cultured for 7, 14 or 21 days, cells were washed with PBS and then digested with $0.25 \%$ trypsin/ETDA. Cells were centrifuged at $200 \mathrm{xg}$ for $5 \mathrm{~min}$ at room temperature, and digested with proteinase K (20 mg/ml; Sigma-Aldrich; Merck KGaA) for $16 \mathrm{~h}$ at $56^{\circ} \mathrm{C}$. After staining with $10 \mu \mathrm{g} / \mathrm{ml}$ Hoechst 33258 dye at room temperature for $10 \mathrm{~min}$ (Sigma-Aldrich; Merck $\mathrm{KGaA}$ ), the DNA content was determined using a fluorescence microplate reader (BioTek Instruments, Inc., Winooski, VT, USA) at $460 \mathrm{~nm}$; calf thymus DNA (Sigma-Aldrich; Merck $\mathrm{KGaA}$ ) was used as a standard and absorption of Hoechst 33258 dye was considered the baseline. The total production 
Table I. Primers for reverse transcription-quantitative polymerase chain reaction.

\begin{tabular}{lll}
\hline Gene & \multicolumn{1}{c}{ Forward primer } & \multicolumn{1}{c}{ Reverse primer } \\
\hline Acan & 5'-GACAAGGACGAGTTCCCTGG-3' & 5'-CTCCGGGGATGTGGCATAAA-3' \\
Sox9 & 5'-TCCAGCAAGAACAAGCCACA-3' & 5'-TCCAGCAAGAACAAGCCACA-3' \\
Col2a1 & 5'-AGATGGCTGGAGGATTTGACG-3' & 5'-TTTCCGGGCTTTCCAGCTT-3' \\
Col1a1 & 5'-GATCCTGCCGATGTCGCTAT-3' \\
RUNX2 & 5'-GTGGCCAGGTTCAACGATCT-3' & 5'-GGGACTTCTTGAGGTTGCCA-3' \\
ENO2 & 5'-TCAAGTCTCCTGCTGACCCT-3' & 5'-TGAGGAATGCGCCCTAAATCA-3' \\
-actin & 5'-CCCATCTATGAGGGTTACGC-3' & 5'-AACGTGTCCTCGGTTTCTCC-3' \\
\hline
\end{tabular}

Acan, aggrecan; Sox9, SRY-box 9; Col2al, collagen type II; Coll al, collagen type I; RUNX2, runt-related transcription factor 2; ENO2, enolase 2.

of GAG was quantified by detecting absorbance at $525 \mathrm{~nm}$, following treatment with $16 \mathrm{mg} / 1$ 1, 9-dimeth-ylmethylene blue (DMMB; Sigma-Aldrich; Merck KGaA) at room temperature for $10 \mathrm{~min}$; chondroitin sulfate (Sigma-Aldrich; Merck $\mathrm{KGaA}$ ) was used as a standard. The GAG content in each cell was normalized to the total DNA content of all cells, which represented its biosynthetic activity under various culture conditions.

Safranin $O$ staining. Safranin O staining was performed to assess the synthesis of GAG. After being fixed with $95 \%$ alcohol for $30 \mathrm{~min}$, at room temperature the cells were stained with $0.1 \%$ safranin O (Sigma-Aldrich; Merck KGaA) for $10 \mathrm{~min}$. Subsequently, the cells were washed with water and sealed with neutral gum. Eventually, cells were observed and images were captured under an inverted phase contrast microscope (Olympus Corporation).

Immunohistochemical staining. Immunohistochemistry was performed to analyze the expression levels of collagen type I $\alpha 1$ chain (COL1A1) and collagen type II $\alpha 1$ chain (COL2A1). After 7, 14 and 21 days, cells were washed three times with PBS, fixed with $95 \%$ alcohol at room temperature for $30 \mathrm{~min}$ and treated with Triton X-100 (Sigma-Aldrich; Merck KGaA) at room temperature for $10 \mathrm{~min}$. To eliminate endogenous peroxidase activity, cells were incubated with $3 \% \mathrm{H}_{2} \mathrm{O}_{2}$ at room temperature for $15 \mathrm{~min}$ and blocked with goat serum (Beijing Solarbio Science \& Technology Co., Ltd.) for $15 \mathrm{~min}$ at room temperature. After incubating with COL1A1 (1:200; Wuhan Boster Biological Technology, Ltd., Wuhan, China) and COL2A1 (BA0533; 1:200; Wuhan Boster Biological Technology Ltd.) primary antibodies overnight at $4^{\circ} \mathrm{C}$, cells were incubated with the secondary antibody (G1080, 1:200; Beijing Solarbio Science \& Technology Co., Ltd.) and followed by biotin-labeled horseradish peroxidase (Zhongshanjinqiao Biotechnology Inc., Wuhan, China) at room temperature for 15 min. A DAB kit (Wuhan Boster Biological Technology, Ltd.) was used to visualize antibody binding. Finally, images of the cells were captured under an inverted phase contrast microscope (Olympus Corporation).

Reverse transcription-quantitative polymerase chain reaction (RT-qPCR) analysis. RT-qPCR was conducted to analyze the expression levels of aggrecan (Acan), SRY-box 9 (Sox9),
Col2a1, Collal, runt-related transcription factor 2 (RUNX2) and enolase 2 (ENO2). The sequences of primers used for RT-qPCR are presented in Table I. Total RNA was extracted using a RNA isolation kit (Wuhan Megentec Biological Technology, Ltd., Wuhan, China), according to the manufacturer's protocol. RT of RNA was performed using a RT kit (Fermentas; Thermo Fisher Scientific, Inc.) and was carried out at $25^{\circ} \mathrm{C}$ for $5 \mathrm{~min}, 42^{\circ} \mathrm{C}$ for $60 \mathrm{~min}$ and $72^{\circ} \mathrm{C}$ for $5 \mathrm{~min}$. RT-qPCR was performed on a qPCR Detection system with Fast Start Universal SYBR Green Master Mix (Roche Diagnostics, Basel, Switzerland) under the following conditions: $10 \mathrm{~min}$ at $95^{\circ} \mathrm{C}$ for initial denaturation, $15 \mathrm{sec}$ at $95^{\circ} \mathrm{C}$ and $1 \mathrm{~min}$ at $60^{\circ} \mathrm{C}$ for final extension (40 cycles). The melting curve data were collected to verify PCR specificity; each gene was analyzed in triplicate. The relative mRNA expression levels were calculated using the $2^{-\Delta \Delta \mathrm{Cq}}(17)$ method with $\beta$-actin as a reliable internal control.

Statistical analysis. Data are presented as the means \pm standard deviation from 4 repeated experiments. Data were analyzed using one-way analysis of variance followed by Dunnett's post hoc test (SPSS version 21; IBM Corp., Armonk, $\mathrm{NY}$, USA). $\mathrm{P}<0.05$ was considered to indicate a statistically significant difference.

\section{Results}

Cytotoxicity of NGF. An MTT assay was performed to detect the cytotoxicity of NGF on BMSCs. Cells were treated with increasing concentrations of $\mathrm{mNGF}(0-0.96 \mu \mathrm{g} / \mathrm{ml})$ or cvNGF $(0-32 \mu \mathrm{g} / \mathrm{ml})$. As shown in Fig. $1, \mathrm{mNGF}$ at concentrations ranging between 0.03 and $0.12 \mu \mathrm{g} / \mathrm{ml}$ and cvNGF at $1.5-16 \mu \mathrm{g} / \mathrm{ml}$ significantly accelerated cell growth $(\mathrm{P}<0.05)$. On the basis of these results, $0.06 \mu \mathrm{g} / \mathrm{ml} \mathrm{mNGF} \mathrm{(Fig.} \mathrm{1A)} \mathrm{and}$ $6 \mu \mathrm{g} / \mathrm{ml} \mathrm{cvNGF}$ (Fig. 1B), which exhibited the most obvious effect on BMSC proliferation, were chosen for further investigation.

Cell viability. To evaluate the effects of NGF on cell viability, a FDA/PI staining kit (Fig. 2) was used. The majority of the cells in all groups were stained green, which indicated a greater amount of viable cells. More viable cells and fewer apoptotic cells (stained red) were detected in the cvNGF group compared with the other groups. 

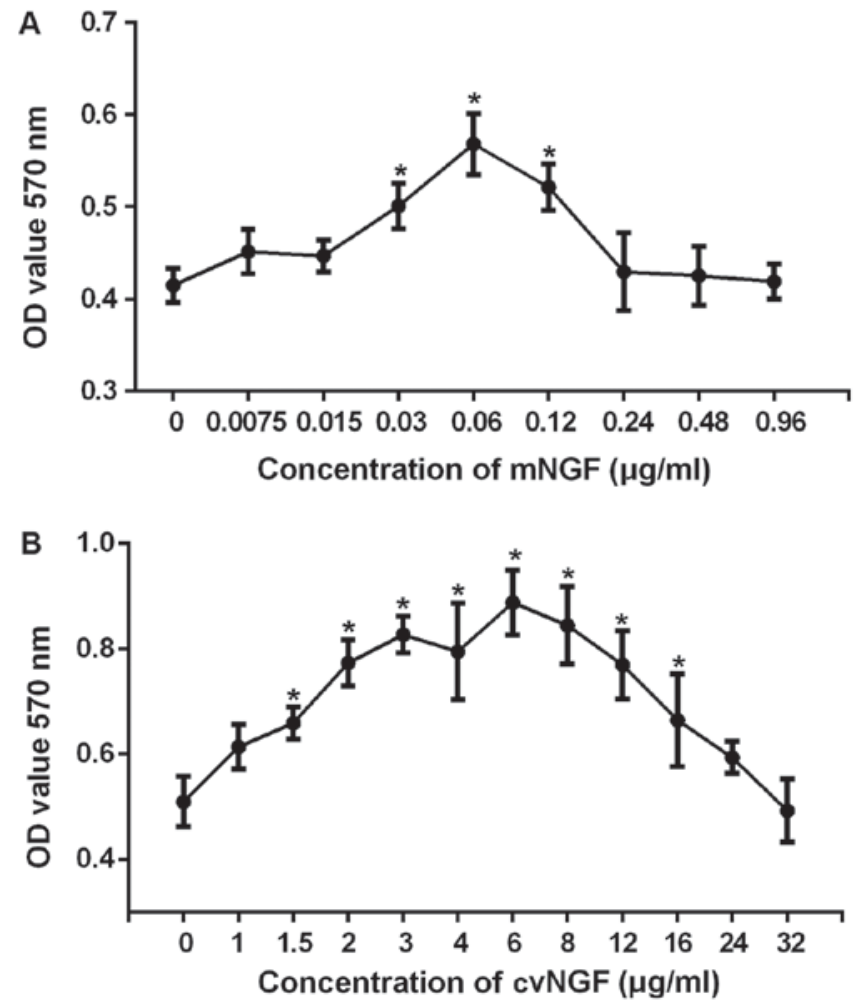

Figure 1. Cytotoxicity of (A) mNGF and (B) cvNGF on bone-derived mesenchymal stem cells following treatment for 3 days. Data are presented as the means \pm standard deviation, $\mathrm{n}=6$. ${ }^{*} \mathrm{P}<0.05$ vs. $0 \mu \mathrm{g} / 1$. cvNGF, cobra-venom-derived NGF; mNGF, murine $\beta$-NGF; NGF, nerve growth factor; OD, optical density.

Cell morphology. Morphological alterations in BMSCs were detected following treatment with NGF for 7, 14 and 21 days using H\&E staining. As shown in Fig. 3, more cells displayed chondrocyte-like morphology in the cvNGF group at the same culture period compared with the control and mNGF groups. In addition, more cell colonies were observed in the cv-NGF group.

Cell proliferation. The proliferation of NGF-treated BMSCs was analyzed according to DNA content. The DNA content in all groups was increased in a time-dependent manner (Fig. 4A). The growth rate of BMSCs was 126 and 59\% higher in the cvNGF and mNGF groups at day 21, respectively, compared with in the control group, as evidenced by the significantly higher DNA content. In particular, cvNGF promoted cell growth compared with the other two groups; these findings were consistent with the results of the cell viability assay (Fig. 2) and H\&E staining (Fig. 3).

GAG secretion. GAG deposition in BMSCs treated with NGFs for 7, 14 and 21 days was measured by DMMB assay and Safranin O staining. As shown in Fig. 4B, GAG production was gradually increased in a time-dependent manner. GAG synthesis in NGF-treated BMSCs was significantly higher compared with in the control group at the same timepoint. Compared with the control group, cvNGF promoted GAG synthesis most effectively among all groups with an increase of $60 \%$ at day 21. Safranin O staining also detected intense staining in the NGF groups compared with in the control group (Fig. 5). In addition, more abundant and homogeneously distributed GAG was detected in BMSCs in the cvNGF group.

Production of collagen type I and type II.Immunohistochemical staining was used to detect the expression of type I and type II collagen in BMSCs following treatment with NGFs in vitro (Fig. 6). Strong type II collagen-positive staining (Fig. 6B) and weaker type I collagen staining (Fig. 6A) were detected in the NGF groups after 7, 14 and 21 days of culturing, particularly in the cvNGF group.

Gene expression. The mRNA expression levels of Acan, Sox9, Col2a1, Colla1, RUNX2 and ENO2 were detected by RT-qPCR. As shown in Fig. 7, cartilage-specific genes, Acan, Sox9 and Col2al, were significantly upregulated by cvNGF compared with the control and mNGF groups. Conversely, Collal was significantly downregulated in the cvNGF group. The expression levels of $R U N X 2$, a specific gene associated with hypertrophy and osteogenic differentiation, were similar to those of Collal. Furthermore, ENO2, a specific gene marker for neural differentiation, was not induced by NGF, as evidenced by reduced expression compared with in the control group.

\section{Discussion}

Articular cartilage repair remains a huge challenge for researchers and clinicians. MSC-based therapies have been employed to tackle these obstacles, due to their high proliferation rate, easy availability and capacity to differentiate into numerous cell types (18). Growth factors are involved in MSC-based therapy. In our previous study, NGF was extracted from Chinese cobra venom using an improved three-step chromatography method, and its specific chondrogenesis-promoting effects on BMSCs were verified (8). Furthermore, cvNGF has been reported to exhibit higher bioactivity compared with other natural sources of NGF $(15,16)$.

The present study focused on comparing the chondrogenic effects of NGF from two sources, commercially purchased recombinant $\mathrm{mNGF}$ and extracted cvNGF, on BMSCs. The results of this study verified that cvNGF greatly accelerated the survival and proliferation of BMSCs compared with the control and mNGF groups, as evidenced by the results of cell viability, morphological and proliferation analyses.

The results of an RT-qPCR analysis demonstrated that the expression levels of Acan, Sox9 and Col2al, which are cartilage specific markers $(19,20)$, were significantly upregulated in the cvNGF group compared with in the other two groups. SOX9 has been reported to act as a chondrogenic transcription factor and is a marker of early cartilage formation that has an important role in the process of chondrogenisis though promoting the production of Acan and activating co-expression with collagen type II $(21,22)$. Consistent with the upregulation of cartilage-specific genes, the deposition of GAG, which serves a pivotal role in maintaining cartilage load-bearing capacity (23), was markedly enhanced by cvNGF, as indicated in the results of a biochemical assay and safranin O staining. In addition, more collagen type II-positive staining was detected in the cvNGF group compared with in the other two groups. These results suggested that cvNGF may 

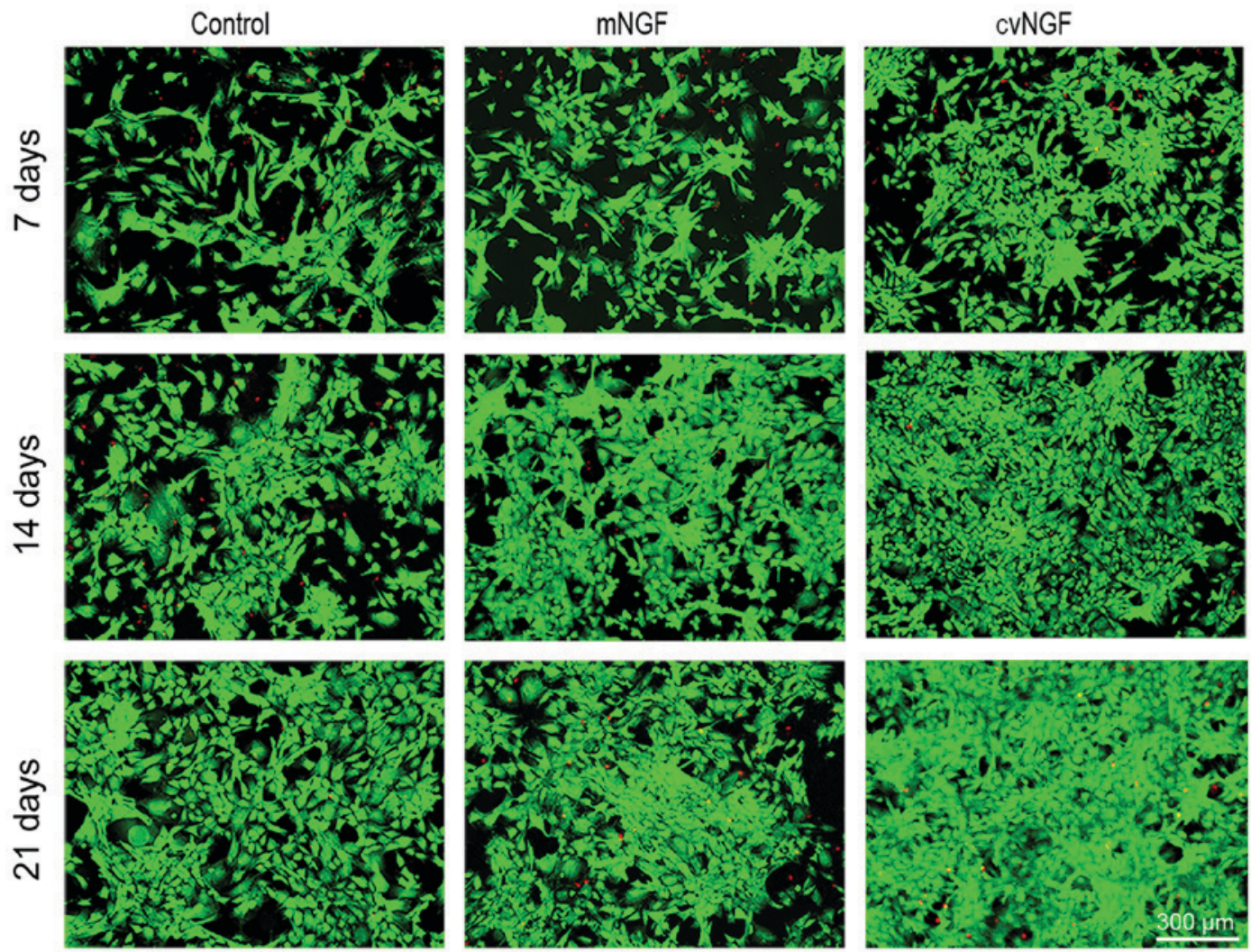

Figure 2. Fluorescein diacetate/propidium iodide staining was used to detect the viability of bone-derived mesenchymal stem cells cultured alone (control) or with NGFs (mNGF, $0.06 \mu \mathrm{g} / \mathrm{ml}$; cvNGF, $6 \mu \mathrm{g} / \mathrm{ml}$ ) for 7, 14 and 21 days. Cell seeding density, $2 \times 10^{4} / \mathrm{ml}$ (original magnification, $\mathrm{x} 100$; scale bar, $300 \mu \mathrm{m}$ ). cvNGF, cobra-venom-derived NGF; mNGF, murine $\beta$-NGF; NGF, nerve growth factor.
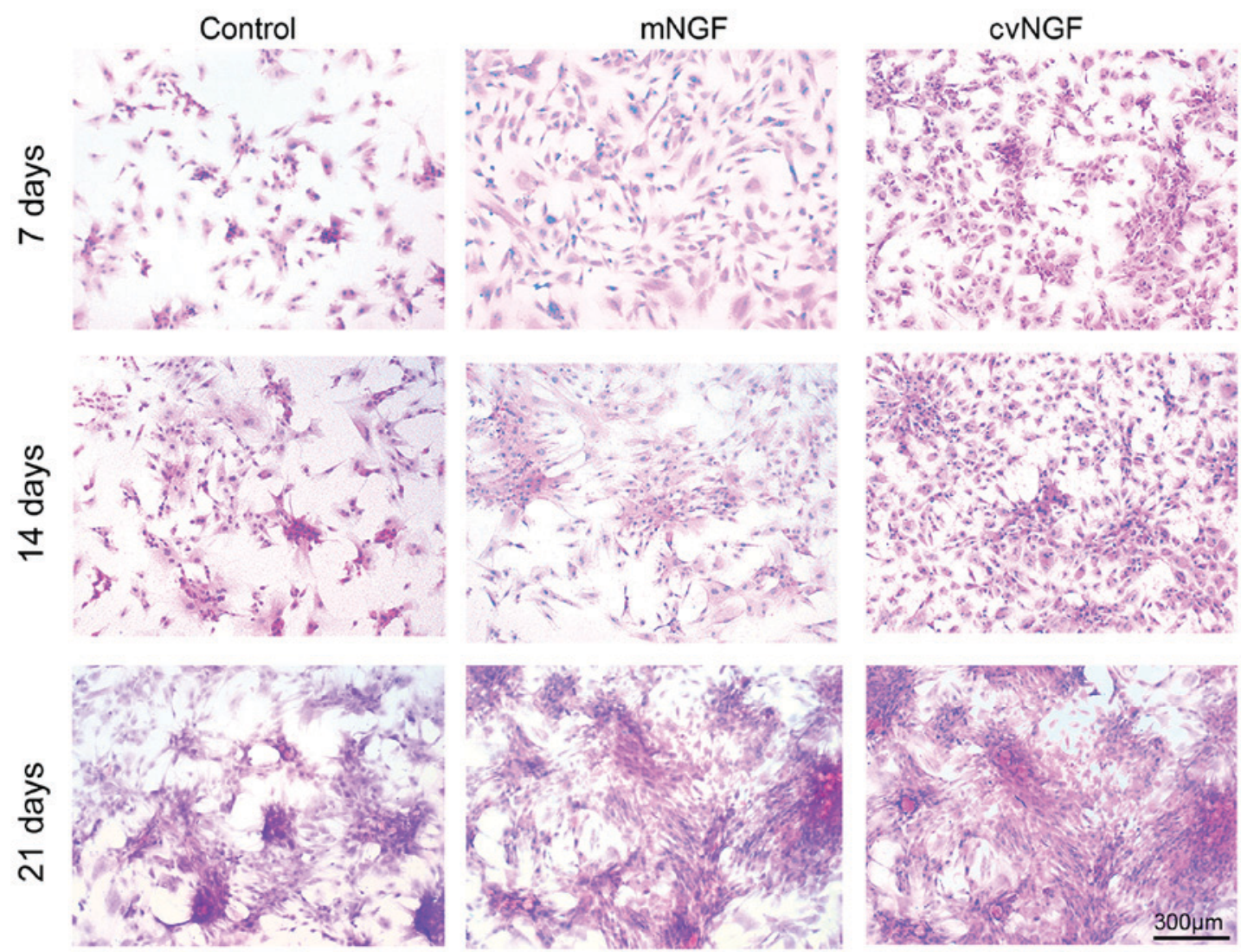

Figure 3. Hematoxylin and eosin staining was used to detect the morphology of bone-derived mesenchymal stem cells cultured alone (control) or with NGFs (mNGF, $0.06 \mu \mathrm{g} / \mathrm{ml}$; cvNGF, $6 \mu \mathrm{g} / \mathrm{ml}$ ) for 7, 14 and 21 days. Cell seeding density, $2 \times 10^{4} / \mathrm{ml}$ (original magnification, $\mathrm{x} 100$; scale bar, $300 \mu \mathrm{m}$ ). cvNGF, cobra-venom-derived NGF; mNGF, murine $\beta$-NGF; NGF, nerve growth factor. 

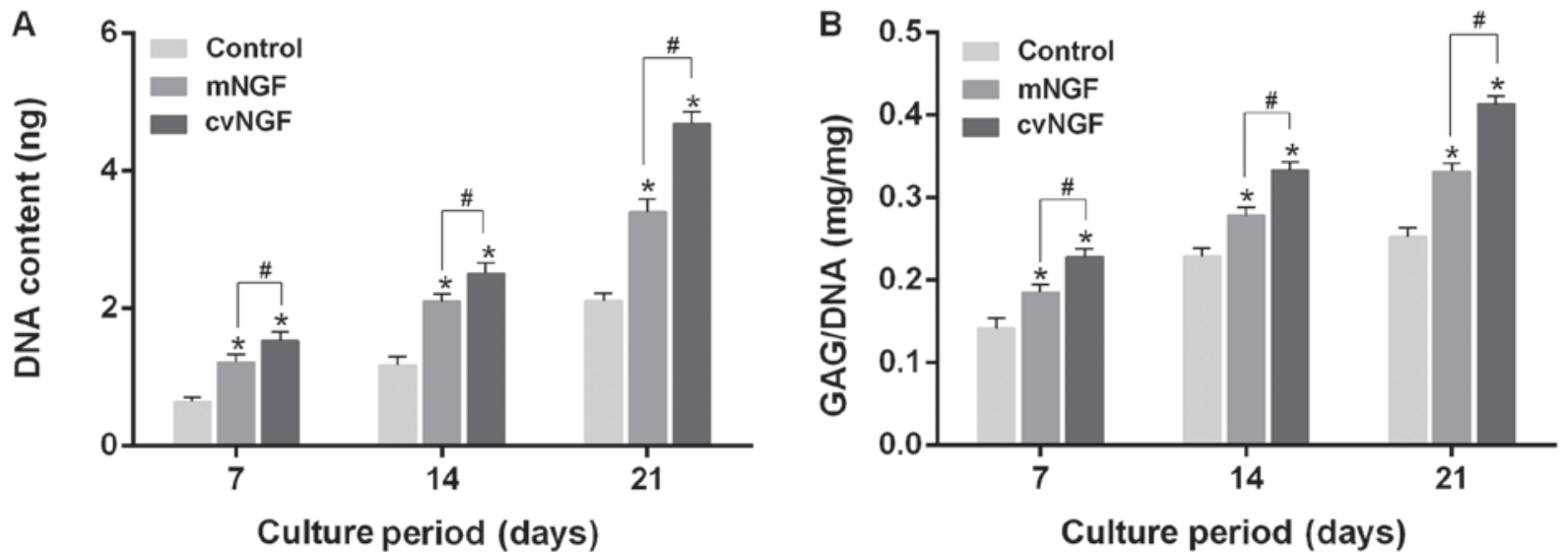

Figure 4. Quantification of cell proliferation, as detected by (A) DNA content and (B) matrix production by GAG. (A) Proliferation of bone-derived mesenchymal stem cells cultured alone (control) or with NGFs (mNGF, $0.06 \mu \mathrm{g} / \mathrm{ml}$; cvNGF, $6 \mu \mathrm{g} / \mathrm{ml}$ ) for 7, 14 and 21 days. (B) GAG (mg) normalized to DNA (mg). Data from four independent experiments were evaluated, and are presented as the means \pm standard deviation. ${ }^{~} \mathrm{P}<0.05$ vs. the control group; ${ }^{\text {}} \mathrm{P}<0.05$, as indicated. cvNGF, cobra-venom-derived NGF; GAG, glycosaminoglycan; mNGF, murine $\beta$-NGF; NGF, nerve growth factor.
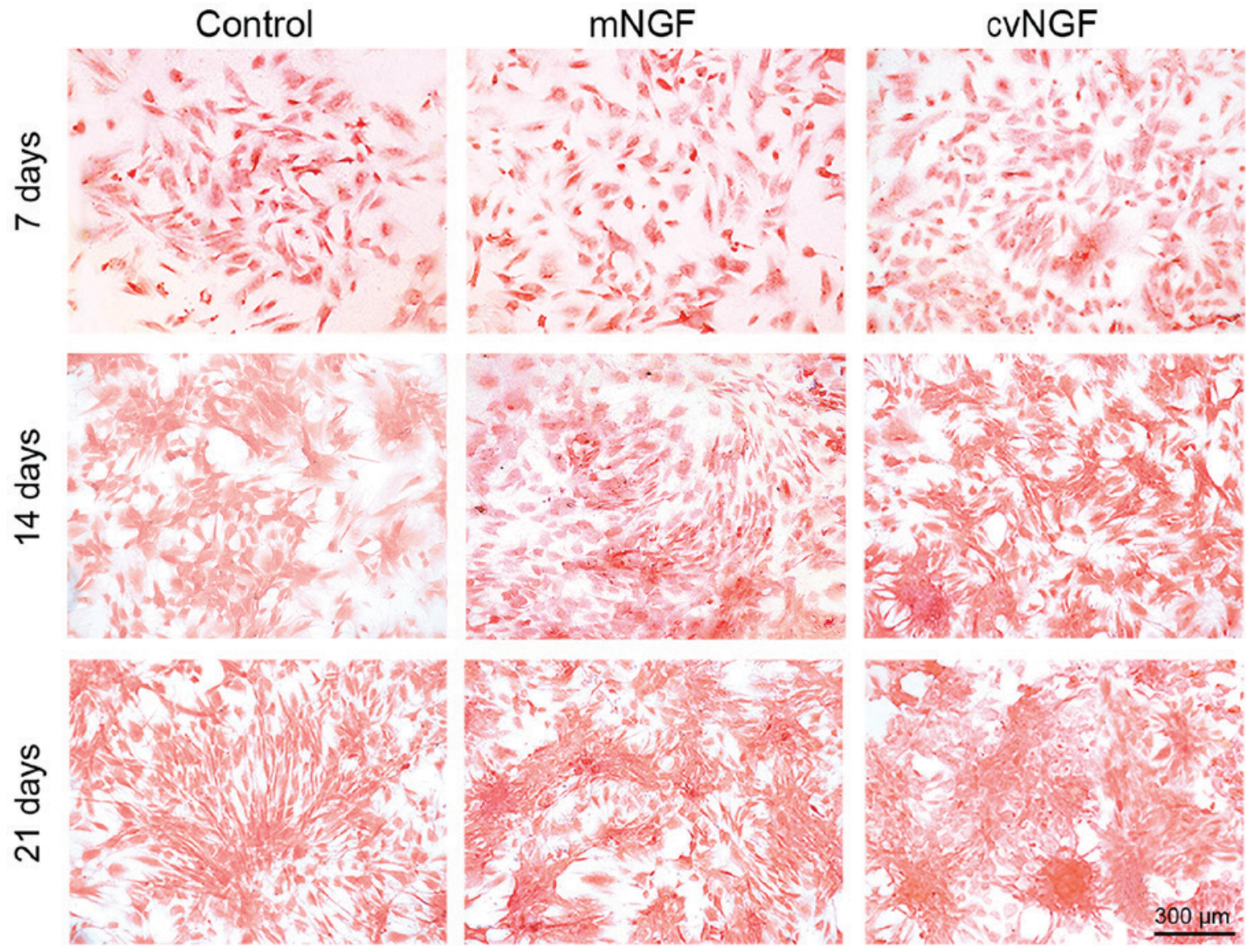

Figure 5. Safranin O staining demonstrated the extracellular matrix synthesis of bone-derived mesenchymal stem cells cultured alone (control) or with NGFs (mNGF, $0.06 \mu \mathrm{g} / \mathrm{ml}$; cvNGF, $6 \mu \mathrm{g} / \mathrm{ml}$ ) for 7, 14 and 21 days. Cell seeding density, 2x104/ml (original magnification, $\mathrm{x} 100$; scale bar, $300 \mu \mathrm{m}$ ). cvNGF, cobra-venom-derived NGF; mNGF, murine $\beta$-NGF; NGF, nerve growth factor.

accelerate chondrocyte proliferation and stimulate cartilage matrix secretion via regulating the key activators of the chondrocyte-specific enhancer, including Acan, Sox9 and Col2al.

Collagen type I is a marker of fibrocartilage (24), which was downregulated in response to treatment with cvNGF, as evidenced by the results of PCR and immunohistochemistry. When type II collagen and cartilage-specific proteoglycan are lost and replaced by a complex collagen phenotype, which is predominately associated with type I collagen and a low level of proteoglycan synthesis, dedifferentiation of chondrocytes ensues. The present study indicated that cvNGF could maintain a phenotype of induced chondrocytes, which was in accord with our previous study that revealed cvNGF protected chondrocytes from differentiation in vitro. Furthermore, 
A
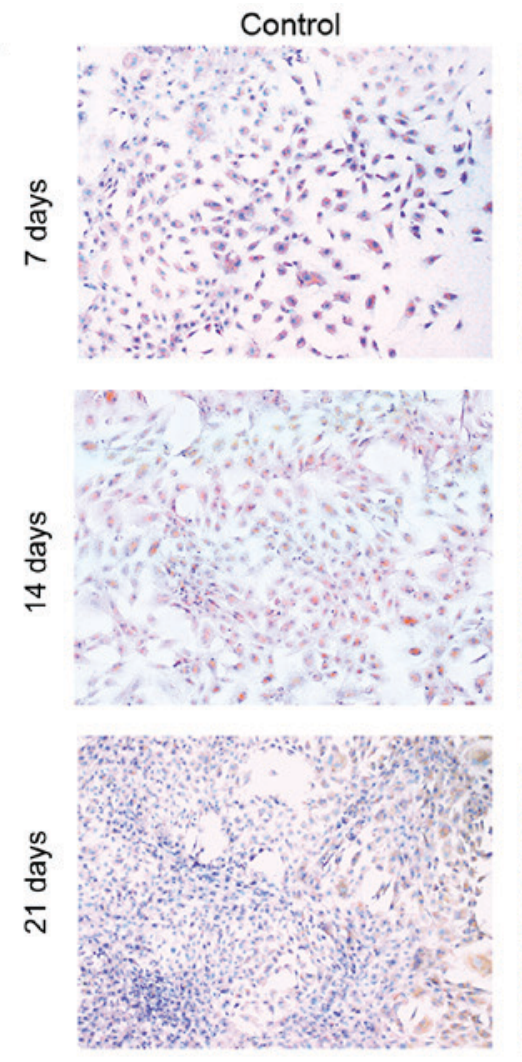

B

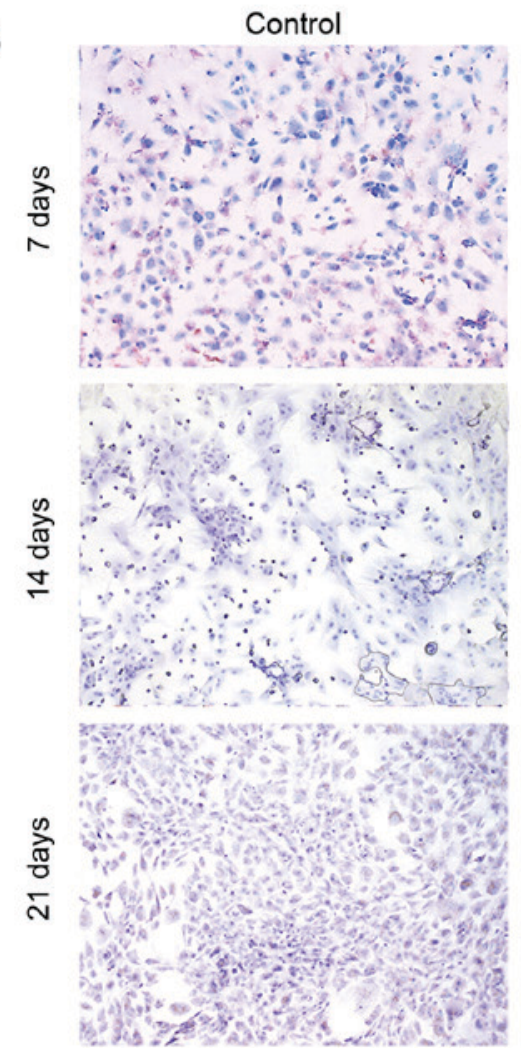

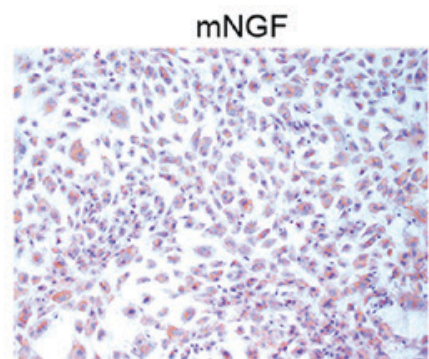
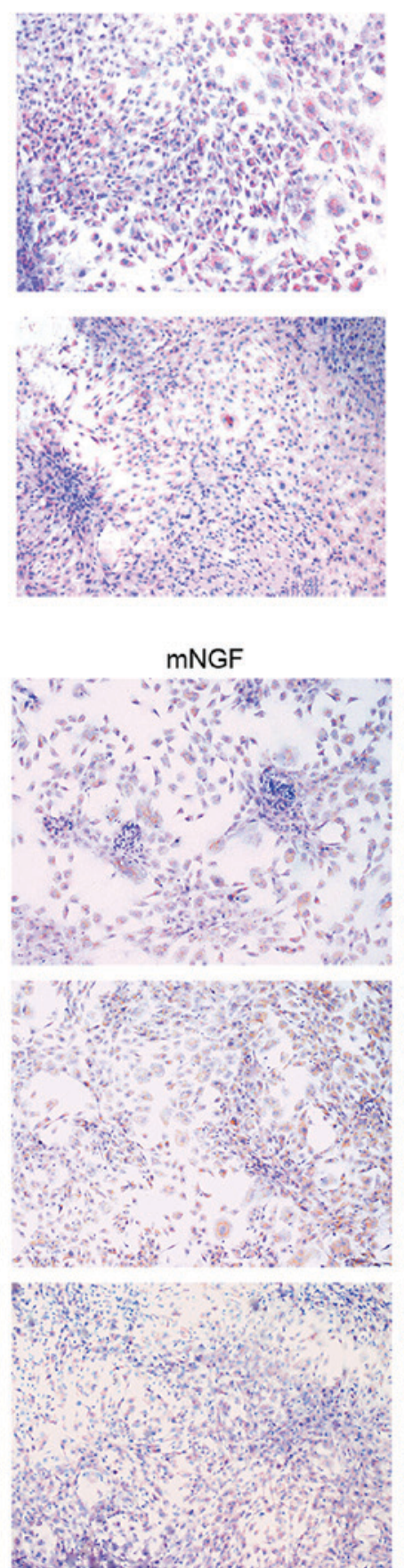
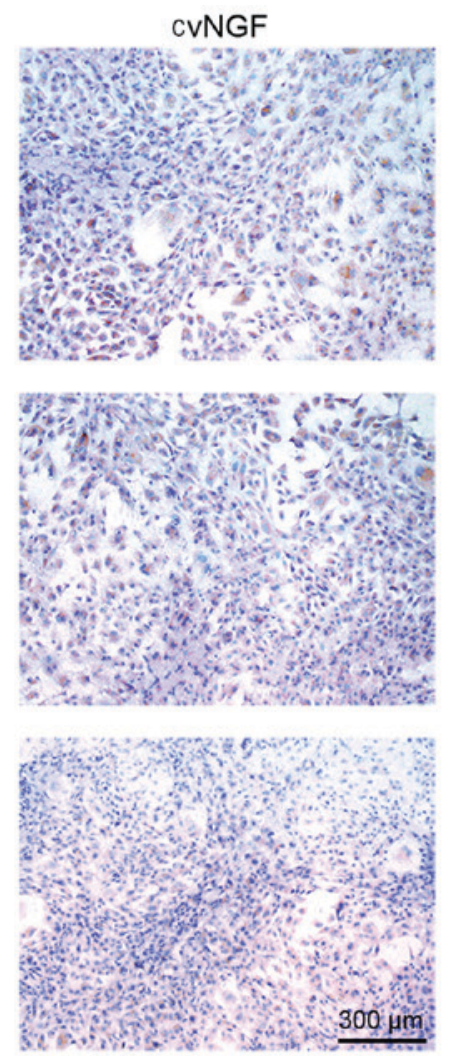

CVNGF

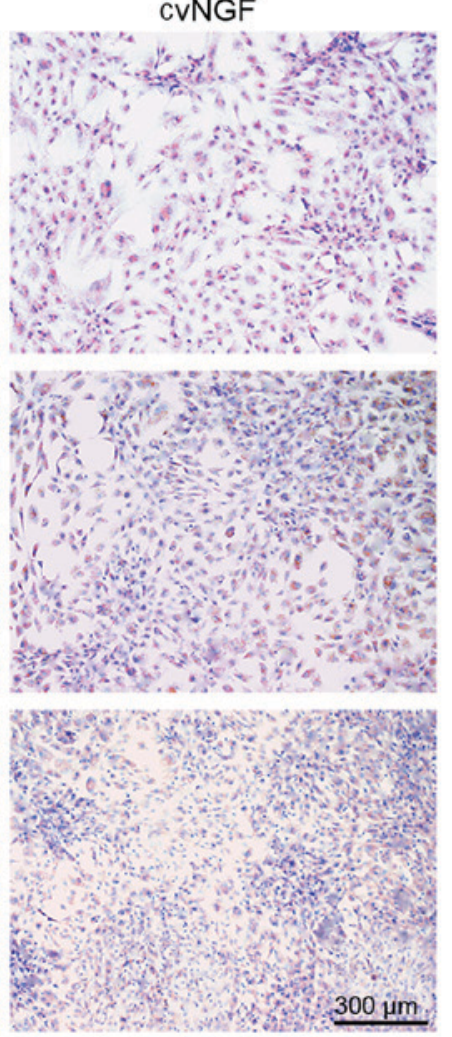

Figure 6. Immunohistochemical staining was used to detect the secretion of (A) type I and (B) type II collagens in bone-derived mesenchymal stem cells cultured alone (control) or with NGFs (mNGF, $0.06 \mu \mathrm{g} / \mathrm{ml}$; cvNGF, $6 \mu \mathrm{g} / \mathrm{ml}$ ) for 7,14 and 21 days. Cell seeding density, $2 \times 10^{4} / \mathrm{ml}$ (original magnification, x100; scale bar, $300 \mu \mathrm{m})$. cvNGF, cobra-venom-derived NGF; mNGF, murine $\beta$-NGF; NGF, nerve growth factor.

$R U N X 2$, a transcription factor for chondrocyte terminal differentiation (25), was also downregulated in the cvNGF group. ENO2, which has a role in neural differentiation, was also suppressed by cvNGF (26) compared with in the mNGF and control groups, thus indicating that cvNGF did not induce BMSCs neural differentiation. 
A

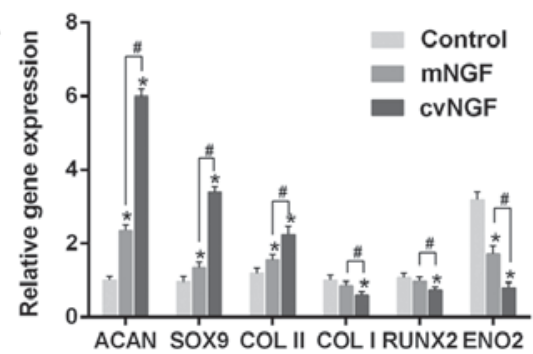

B

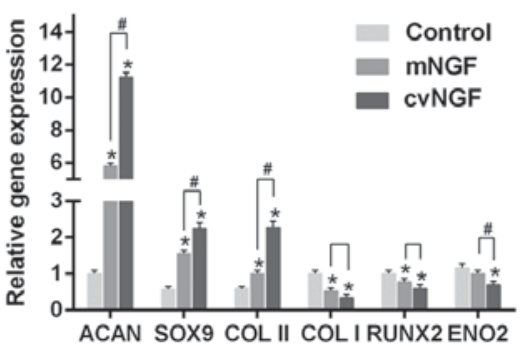

C

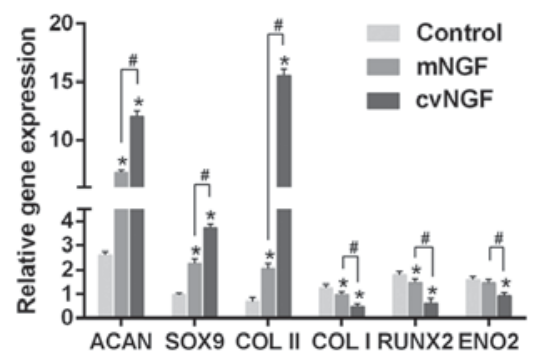

Figure 7. Quantitative comparison of Acan, Sox9, Col2a1, Colla1, RUNX2 and ENO2 mRNA expression, as detected by reverse transcription-quantitative polymerase chain reaction. Bone-derived mesenchymal stem cells cultured alone (control) or with NGFs (mNGF, $0.06 \mu \mathrm{g} / \mathrm{ml}$; cvNGF, $6 \mu \mathrm{g} / \mathrm{ml}$ ) for (A) 7 , (B) 14 and (C) 21 days. Data are presented as the means \pm standard deviation from 3 repeated experiments. The mRNA expression levels were analyzed using the $2^{-\Delta \Delta \mathrm{Cq}}$ method, with $\beta$-actin as the internal control. ${ }^{*} \mathrm{P}<0.05$ vs. the control group; ${ }^{\#} \mathrm{P}<0.05$, as indicated. Acan, aggrecan; Col2al, collagen type II; Colla1, collagen type I; cvNGF, cobra-venom-derived NGF; ENO2, enolase 2; mNGF, murine $\beta$-NGF; NGF, nerve growth factor; RUNX2, runt-related transcription factor 2; Sox 9 , SRY-box 9.

The present results indicated that $6 \mu \mathrm{g} / \mathrm{ml} \mathrm{cvNGF}$ and $0.06 \mu \mathrm{g} / \mathrm{ml} \mathrm{mNGF}$ resulted in increased cell proliferation. Although the effective concentration of mNGF was lower than that of cvNGF, the chondrogenic effects of cvNGF on BMSCs were superior to mNGF. Furthermore, the high cost and sophisticated manufacturing process of mNGF limits its clinical application. Conversely, cvNGF is separated from natural venom, which is easily accessed and low cost, and is therefore conducive to clinical use. The present results suggested that cvNGF may be a promising growth factor for cartilage reconstruction.

In conclusion, the present study compared the chondrogenic effects of NGF from two sources, the commercially purchased mNGF and the extracted cvNGF, on BMSCs in vitro. The results suggested that cvNGF was more effective at inducing chondrogenic differentiation of BMSCs compared with mNGF. The easy acquirement and low cost of cvNGF indicated that it may be considered a favorable growth factor for cartilage reconstruction and beneficial for clinical applications. However, the present study conducted only in vitro experiments; therefore, these findings need to be further confirmed in vivo.

\section{Acknowledgements}

Not applicable

\section{Funding}

The National Natural Science Fund of China (grant nos. 81760326 and 81460345), the Guangxi Science and Technology Major Project (grant no. Guike AA17204085), the Distinguished Young Scholars Program of Guangxi Medical University and the Key Scientific Research Collaboration Program of Guangxi Biomedical Collaborative Innovation Center (grant no. GCICB-SR-2017002), the 2018 Basic Ability Improvement Project for Middle-aged and Young Teachers in Colleges and Universities in Guangxi and the 2017 Young Creative Talents Training Plan of Guangxi Collaborative Innovation Center of Biomedicine (grant no. GCICB-TC-2017005).

\section{Availability of data and materials}

All data generated or analyzed during this study are included in this published article.

\section{Authors' contributions}

LZ and JZ designed the study and directed its structure. ZM and $\mathrm{ZL}$ performed the experiments and contributed to the writing and revision of the manuscript. SL contributed to the revision of the manuscript, performed the PCR and processed the data. DL, YH and HW were responsible for the statistical analysis.

\section{Ethics approval and consent to participate}

All experiments were conducted in accordance with the standard guidelines approved by the Animal Care and Experiment Committee of Guangxi Medical University (Guangxi, China; protocol number: 2014-12-3), and the present study was approved by the ethics committee of Guangxi Medical University.

\section{Patient consent for publication}

Not applicable.

\section{Competing interests}

The authors declare that they have no competing interests.

\section{References}

1. Risbud MV and Sittinger M: Tissue engineering: Advances in in vitro cartilage generation. Trends Biotechnol 20: 351-356, 2002.

2. Wu YN, Yang Z, Hui JH, Ouyang HW and Lee EH: Cartilaginous ECM component-modification of the micro-bead culture system for chondrogenic differentiation of mesenchymal stem cells. Biomaterials 28: 4056-4067, 2007.

3. Nakasa T and Ochi M: Cell based therapy for articular cartilage injury. Clin Calcium 21: 890-895, 2011 (In Japanese).

4. Caldwell KL and Wang J: Cell-based articular cartilage repair: The link between development and regeneration. Osteoarthritis Cartilage 23: 351-362, 2015.

5. Jiang T, Xu G, Wang Q, Yang L, Zheng L, Zhao J and Zhang X: In vitro expansion impaired the stemness of early passage mesenchymal stem cells for treatment of cartilage defects. Cell Death Dis 8: e2851, 2017.

6. Szychlinska MA, Stoddart MJ, D'Amora U, Ambrosio L, Alini M and Musumeci G: Mesenchymal stem cell-based cartilage regeneration approach and cell senescence: Can we manipulate cell aging and function? Tissue Eng Part B Rev 23: 529-539, 2017. 
7. Raghunath J, Salacinski HJ, Sales KM, Butler PE and Seifalian AM: Advancing cartilage tissue engineering: The application of stem cell technology. Curr Opin Biotechnol 16: 503-509, 2005

8. Lu Z, Lei D, Jiang T, Yang L, Zheng L and Zhao J: Nerve growth factor from Chinese cobra venom stimulates chondrogenic differentiation of mesenchymal stem cells. Cell Death Dis 8: e2801, 2017.

9. van Beuningen HM, Glansbeek HL, van der Kraan PM and van den Berg WB: Differential effects of local application of BMP-2 or TGF-beta 1 on both articular cartilage composition and osteophyte formation. Osteoarthritis Cartilage 6: 306-317, 1998.

10. Bakker AC, van de Loo FA, van Beuningen HM, Sime P, van Lent PL, van der Kraan PM, Richards CD and van den Berg WB: Overexpression of active TGF-beta-1 in the murine knee joint: Evidence for synovial-layer-dependent chondro-osteophyte formation. Osteoarthritis Cartilage 9: 128-136, 2001.

11. Blaney Davidson EN, Vitters EL, van Beuningen HM, van de Loo FA, van den Berg WB and van der Kraan PM: Resemblance of osteophytes in experimental osteoarthritis to transforming growth factor beta-induced osteophytes: Limited role of bone morphogenetic protein in early osteoarthritic osteophyte formation. Arthritis Rheum 56: 4065-4073, 2007.

12. Li C, Jiang J, Zheng Z, Lee KS, Zhou Y, Chen E, Culiat CT, Qiao Y, Chen X, Ting K, et al: Neural EGFL-like 1 is a downstream regulator of runt-related transcription factor 2 in chondrogenic differentiation and maturation. Am J Pathol 187: 963-972, 2017.

13. Grässel SG: The role of peripheral nerve fibers and their neurotransmitters in cartilage and bone physiology and pathophysiology. Arthritis Res Ther 16: 485, 2014.

14. Nojiri Y, Takeda S, Enomoto M, Nishitsuji H, Masuda T, Sotome S and Shinomiya K: Co-overexpression of GDNF and GFRalpha1 induces neural differentiation in neural progenitor cells in comparison to bone marrow stromal cells. J Med Dent Sci 55: 121-128, 2008.

15. Lipps BV: Detection of nerve growth factor (NGF) in venoms from diverse source: Isolation and characterization of NGF from the venom of honey bee (Apis melifera). J Nat Toxins 9: 13-19, 2000 .
16. Lipps BV: Isolation of nerve growth factor (NGF) from human body fluids; saliva, serum and urine: Comparison between cobra venom and cobra serum NGF. J Nat Toxins 9: 349-356, 2000

17. Livak KJ and Schmittgen TD: Analysis of relative gene expression data using real-time quantitative PCR and the 2(-Delta Delta C(T)) method. Methods 25: 402-408, 2001.

18. Caplan AI: Adult mesenchymal stem cells for tissue engineering versus regenerative medicine. J Cell Physiol 213: 341-347, 2007.

19. Tew SR, Li Y, Pothacharoen P, Tweats LM, Hawkins RE and Hardingham TE: Retroviral transduction with SOX9 enhances re-expression of the chondrocyte phenotype in passaged osteoarthritic human articular chondrocytes. Osteoarthritis Cartilage 13: 80-89, 2005.

20. Uebersax L, Merkle HP and Meinel L: Insulin-like growth factor I releasing silk fibroin scaffolds induce chondrogenic differentiation of human mesenchymal stem cells. J Control Release 127: $12-21,2008$.

21. Marshall OJ and Harley VR: Molecular mechanisms of SOX9 action. Mol Genet Metab 71: 455-462, 2000.

22. Tew SR and Clegg PD: Analysis of post transcriptional regulation of SOX9 mRNA during in vitro chondrogenesis. Tissue Eng Part A 17: 1801-1807, 2011 .

23. Robinson D, Ash H, Yayon A, Nevo Z and Aviezer D Characteristics of cartilage biopsies used for autologous chondrocytes transplantation. Cell Transplant 10: 203-208, 2001.

24. Zhang W, Chen J, Tao J, Jiang Y, Hu C, Huang L, Ji J and Ouyang HW: The use of type 1 collagen scaffold containing stromal cell-derived factor-1 to create a matrix environment conducive to partial-thickness cartilage defects repair. Biomaterials 34: 713-723, 2013.

25. Liu CF, Samsa WE, Zhou G and Lefebvre V: Transcriptional control of chondrocyte specification and differentiation. Semin Cell Dev Biol 62: 34-49, 2017.

26. Yoshii SR, Kuma A and Mizushima N: Transgenic rescue of Atg5-null mice from neonatal lethality with neuron-specific expression of ATG5: Systemic analysis of adult Atg5-deficient mice. Autophagy 13: 763-764, 2017.

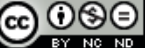

$$
\begin{aligned}
& \text { This work is licensed under a Creative Commons } \\
& \text { Attribution-NonCommercial-NoDerivatives } 4.0 \\
& \text { International (CC BY-NC-ND 4.0) License. }
\end{aligned}
$$

